
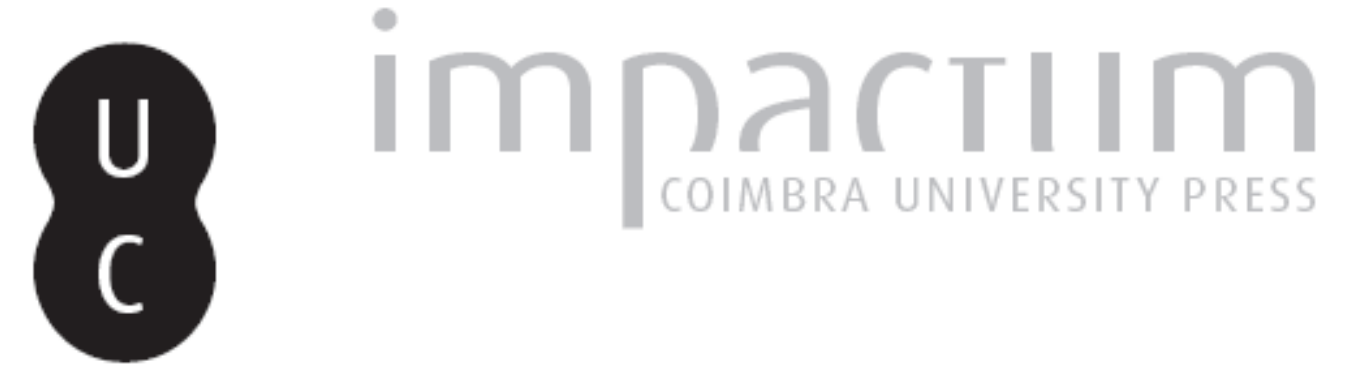

\title{
La concomittance de la nature des êtres et du temps selon l'Ambiguum 10 de Maxime le Confesseur: une trace byzantine de l'exegese tardo-antique du Timee
}
Autor(es):
Mueller-Jourdan, Pascal

Publicado por: Imprensa da Universidade de Coimbra

URL persistente:

URI:http://hdl.handle.net/10316.2/42230

DOI:

DOI:https://doi.org/10.14195/2183-4105_6_6

Accessed : $\quad$ 26-Apr-2023 05:08:13

A navegação consulta e descarregamento dos títulos inseridos nas Bibliotecas Digitais UC Digitalis, UC Pombalina e UC Impactum, pressupõem a aceitação plena e sem reservas dos Termos e Condições de Uso destas Bibliotecas Digitais, disponíveis em https://digitalis.uc.pt/pt-pt/termos.

Conforme exposto nos referidos Termos e Condições de Uso, o descarregamento de títulos de acesso restrito requer uma licença válida de autorização devendo o utilizador aceder ao(s) documento(s) a partir de um endereço de IP da instituição detentora da supramencionada licença.

Ao utilizador é apenas permitido o descarregamento para uso pessoal, pelo que o emprego do(s) título(s) descarregado(s) para outro fim, designadamente comercial, carece de autorização do respetivo autor ou editor da obra.

Na medida em que todas as obras da UC Digitalis se encontram protegidas pelo Código do Direito de Autor e Direitos Conexos e demais legislação aplicável, toda a cópia, parcial ou total, deste documento, nos casos em que é legalmente admitida, deverá conter ou fazer-se acompanhar por este aviso.

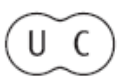




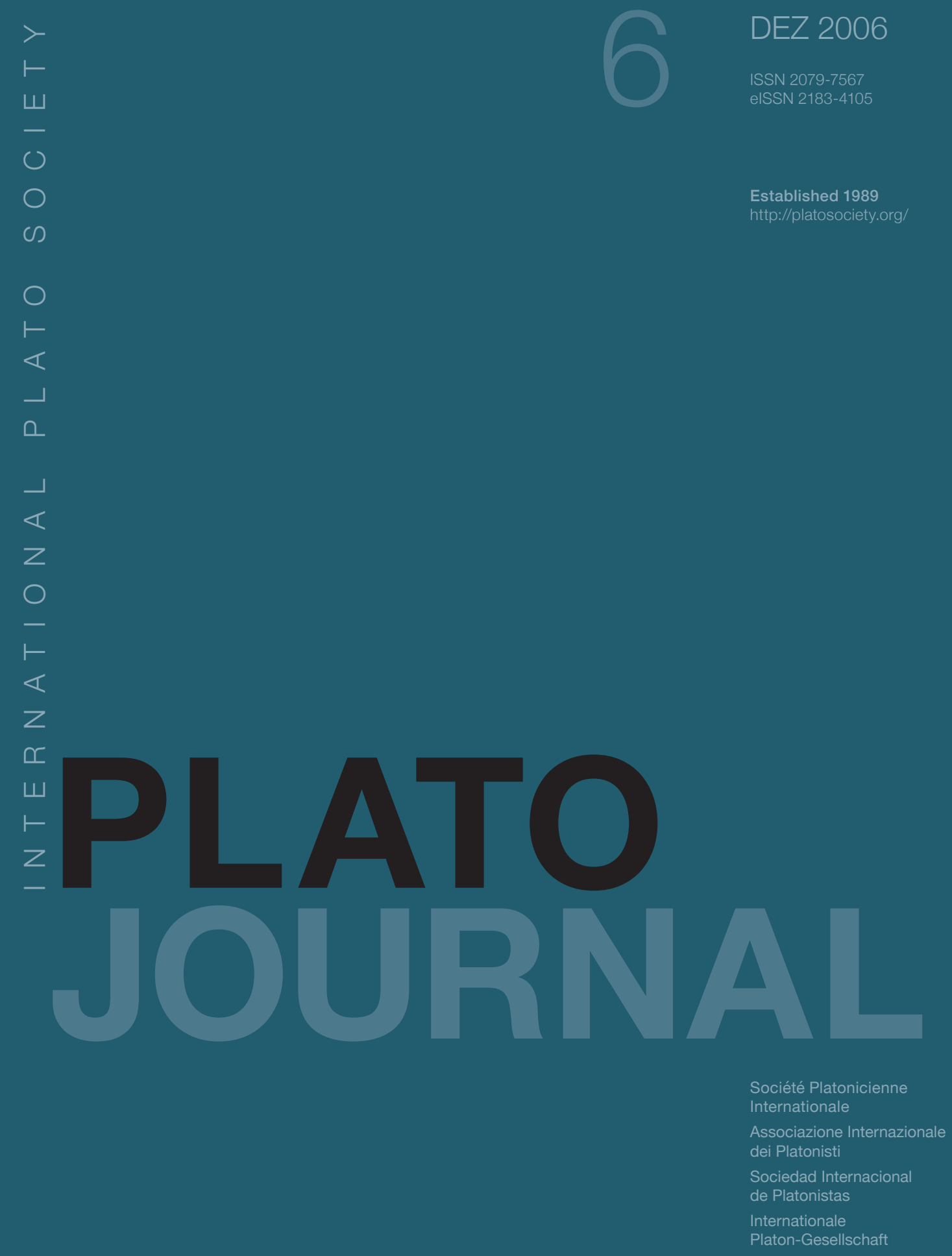


Il est assez fréquent d'observer dans la littérature théologique byzantine d'importantes traces de problèmes philosophiques remontant à la plus haute Antiquité, probables résurgences d'un fond de culture tardo-antique devenu commun au christianisme et au paganisme. Il n'est pas rare non plus qu'on ne décèle qu'avec peine l'origine parfois technique des questions philosophiques initiales. Tel pourrait être le cas d'une affirmation de Maxime le Confesseur, moine spéculatif du VII ${ }^{\text {ème }}$ siècle byzantin, introduisant une interprétation symbolique du récit évangélique de la Transfiguration. ${ }^{1}$

[Theôria sur Moïse et Elie: $\Theta^{‘}$ ]

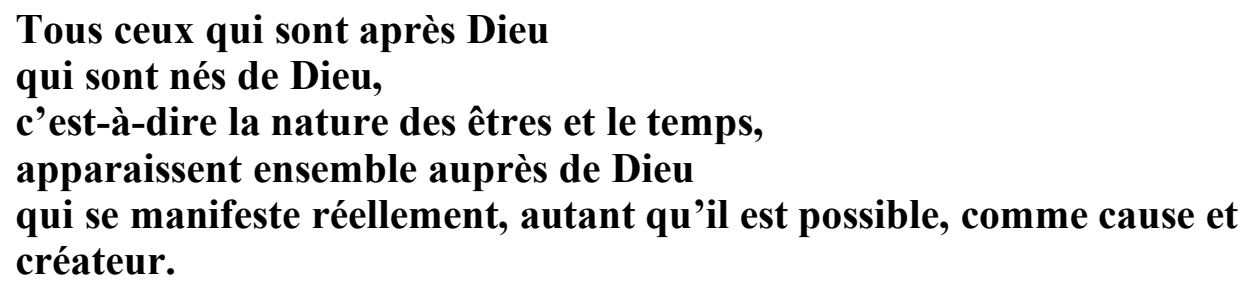

De ces deux, [B] Moïse serait 'type du temps', non seulement comme celui qui enseigne le temps et le nombre qui lui correspond (ce Moïse en effet fut le premier à nombrer le temps d'après la genèse du monde) ou comme celui qui introduisit un culte inscrit dans le temps mais aussi comme celui qui ne devait pas entrer corporellement dans le repos final avec ceux-là même qu'il avait charge d'instruire avant l'annonce de la divine Bonne Nouvelle.

Le temps n'est-il pas en effet aussi de cette sorte ? Ne devançant, ni ne se mouvant avec ceux qu'il avait naturellement charge d'escorter jusqu'à la vie divine de l'aiôn à venir. Il saisit en effet Jésus qui est le successeur de tout, et du temps et de l'aiôn même si par ailleurs les logoi du temps demeurent en Dieu comme le montre sous voile ( $\mu v \sigma \tau 1 \kappa \omega \tilde{s})$ l'entrée de la loi dans le désert, loi donnée par Moïse à ceux qui ont reçu la terre en possession.

Le temps en effet est aiôn quand le mouvement est arrêté et l'aiôn est temps quand, $[\boldsymbol{C}]$ porté par le mouvement, il est mesuré. De sorte que je dis, pour le saisir en une définition, que l'aiôn est temps privé de mouvement et que le temps est aiôn mesuré par du mouvement.

Quant à Elie, il serait 'type de la nature', non seulement pour avoir gardé inaltérés les logoi de sa propre nature et être resté libre, pour ce qui les concerne et selon l'opinion, de pensées instables issues des passions, mais aussi pour avoir éduqué à juger, par exemple 'ce qu'est la loi naturelle', ceux qui font un usage contre-nature de la nature. Car la nature même est telle qu'elle sanctionne autant ceux qui entreprennent de la corrompre que ceux qui s'exercent à vivre contre-nature. Elle les sanctionne par le fait

\footnotetext{
1 Pour rappel, il existe trois récits évangéliques de la Transfiguration : Matthieu 17.1-8 ; Marc 9.2-8 ; Luc 9.28-36.
} 
qu'ils ne possèdent plus naturellement la pleine puissance de la nature même, étant déjà réduits dans leur intégrité naturelle et par là sanctionnés dans la mesure où, inconsciemment et de façon irréfléchie, [D] ils se donnent à eux-mêmes une carence d'être par inclinaison vers le non-être.

Ambiguum 10, PG 91, 1164A-D ${ }^{2}$

...font suite à ce texte deux comparaisons, l'une entre Moïse et le monde sensible dans la mesure où la naissance et la mort du prophète sont un fait avéré et nous sont connues et l'autre entre Elie et le monde intelligible car il se trouve que l'histoire biblique ne dit rien de la naissance d'Elie, ni même si elle a eu lieu, ni si sa fin (le char de feu) ${ }^{3}$ fut suivie de mort et de corruption.

Bien que ce texte et sa thématique soient denses et parsemés d'importantes difficultés qui mériteraient un traitement approfondi, nous voudrions limiter le propos de cette notice à l'axiome qui l'introduit: Tous ceux qui sont après Dieu, qui sont nés de Dieu, c'est-à-dire la nature des êtres et le temps, apparaissent ensemble

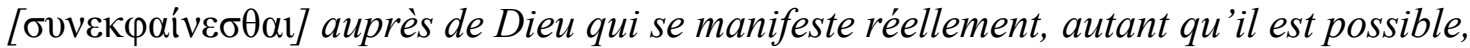
comme cause et créateur.

L'apparition de Moïse et d'Elie entourant le Christ transfiguré représenterait, selon le moine érudit, le 'type' du temps pour Moïse et le 'type' de la nature des êtres pour Elie révélés ensemble auprès du Verbe Démiurge. ${ }^{4}$ La trilogie 'démiurge / nature des êtres / temps' que nous nous proposons de brièvement analyser dans son acclimatation byzantine n'est pas sans rappeler quelques lignes du Timée qui rapporte une configuration similaire, semble-t-il largement admise à l'époque de Platon et qui pourrait s'appuyer - comme le mentionne Timée au début de son exposé- sur la foi d'hommes de sens $^{5}$ : la concomitance du Ciel (ou monde) ${ }^{6}$ et du temps. ${ }^{7}$

Il ne nous revient pas ici d'analyser dans le détail les raisons de cet 'arrangement' qui dans le Timée lui-même semble s'imposer et qui paraît assez claire à première lecture mais plutôt de tenter d'observer ce qu'il va devenir dans l'ébauche d'une théologie byzantine du monde créé dans un environnement de pensée influencé par la philosophie tardo-antique. L'objectif de nos remarques se limitera donc à identifier quelques éléments concernant l'assimilation chrétienne de ce point de cosmologie antique.

Rompu à la pratique de l'exégèse symbolique des textes des Saintes Ecritures, Maxime le Confesseur trahit cependant une bonne familiarité avec les problèmes

\footnotetext{
${ }^{2}$ On trouvera une bonne traduction anglaise de ce texte réalisée par A.Louth (in : Maximus the Confessor, London, New York, 1996), une bonne traduction italienne par Cl. Moreschini (in : Massimo il Confessore. Ambigua, Milano, Bompiani, 2003) et une traduction française faite par E.Ponsoye (à utiliser avec la plus grande précaution).

3 Voir : 2 Rois 2.11 ss.

4 L'expression est de Maxime. Cf., Maxime Le Confesseur, Ambiguum 10, 1164d, PG 91.

5 Cf., le début du discours de Timée, in : Platon, Timée [29e], A.Rivaud ed.

${ }^{6}$ L'instabilité du vocabulaire utilisé jusque chez les commentateurs tardo-antique du Timée (cf., Proclus, In Platonis Timaeum Commentaria [100.3-4], E.Diehl ed.) proviendrait de Platon lui-même : "Soit le ciel dans son ensemble ou le monde -s'il arrive qu'un autre nom lui convienne mieux, donnonslui ce nom», in : Platon, Timée [28b], traduction L.Brisson.

${ }^{7}$ Cf., la reprise résumée de la section où Timée expose la naissance du Temps : "Le temps est donc né en même temps que le ciel afin que, engendrés en même temps, ils soient dissous en même temps, si jamais ils doivent connaître la dissolution » [Timée 38b].
} 
discutés dans les Ecoles philosophiques de l'Antiquité tardive. ${ }^{8}$ La principale difficulté concernant son style provient du fait que Maxime n'est pas un compilateur à l'instar de Jean Damascène mais un 'reformulant'. Retrouver les théories philosophiques qu'ils convoquent dans son propre système devient par conséquent singulièrement difficile à entreprendre mais pas tout à fait impossible.

À l'instar de plusieurs de ses prédécesseurs chrétiens, ${ }^{9}$ Maxime introduit en maints endroits de sa propre œuvre théologique des considérations de nature philosophique sur la constitution du monde. Bien qu'il ne nous offre aucun traité systématique de cosmologie, le savant moine traite à plusieurs reprises de questions touchant à l'origine des choses, à leur fin, au mouvement, au lieu, au temps et à la Providence etc. Il n'est donc guère étonnant de retrouver chez le savant byzantin certains lieux communs propres au platonisme tardo-antique intégrés à une vision originale du monde qui connaîtra une postérité balayant les rivages du XXI ${ }^{\text {ème }}$ siècle dans son adaptation à la pensée religieuse russe et à la théologie de l'Eglise d'Orient des $\mathrm{XIX}^{\text {ème }}$ siècle et $\mathrm{XX}^{\text {eme }}{ }^{10}$

Les idées convoquées pour l'exégèse allégorique du récit de la Transfiguration

L'interprétation symbolique de Maxime veut donc voir dans la présence de Moïse au côté du Christ le 'type du temps'. Maxime en donne deux raisons. La première s'appuie sur le fait que Moïse est, pour l'Antiquité, l'auteur du récit de la Genèse. ${ }^{11}$ Ce récit expose non seulement les conditions et les étapes de la naissance du monde mais dévoile également l'institution du temps. Moïse -premier à compter le temps relativement à la genèse du monde- enseigna, aux dires de Maxime, non seulement la notion de 'temps' mais également le nombre qui lui correspond. La deuxième s'enracine dans le fait que Moïse fut l'instigateur d'un culte inscrit dans le temps. De cette explicitation allégorique d'un Moïse 'type du temps', Maxime va tirer parti pour exposer quelques-unes de ses lignes les plus saillantes d'une conception, finalement assez traditionnelle, du temps. ${ }^{12}$

\footnotetext{
${ }^{8}$ Il faut signaler que la plupart des études maximiennes, même celles qui comptent parmi les plus récentes, peinent à envisager une lecture 'philosophique' du chrétien byzantin. Deux contre-exemples méritent toutefois d'être signalés, voir : St.GERSH, From Iamblichus to Eriugena. An Investigation of the Prehistory and Evolution of the Pseudo-Dionysian Tradition, Leiden, Brill, 1978, mais également : T.Tollefsen, The Christocentric Cosmology of St. Maximus the Confessor. A Study of his Metaphysical Principles, Universitet i Oslo, Acta humaniora nr. 72, 2000.

9 Voir par exemple : COSMAS InDICOPLEUSTES, Topographie chrétienne, 3 T., W. Wolska-Conus ed., Paris, Cerf, SC 141, 159, 197, 1968-1970-1973; W.WOLSKA, Recherche sur la 'Topographie chrétienne' de Cosmas Indicopleustès. Théologie et science au VI ${ }^{\mathrm{ème}}$ siècle, Paris, PUF, 1962 ; JEAN PhILOPOn, De opificio mundi, W.Reichardt ed., Leipzig, Teubner, 1897 ; Cl.SCHOLTEN, Antike Naturphilosophie und christliche Kosmologie in der Schrift "De opificio mundi" des Johannes Philoponos, Berlin - New York, Walter De Gruyter, 1996.

10 Voir par exemple, les travaux de Vladimir Lossky (Essai sur la théologie mystique de l'Eglise d'Orient, Paris, Cerf, 1990 [réédition]), John Meyendorff (Byzantine theology: historical trends and doctrinal themes, New York, Fordham University Press, $1983^{2}$ ) et Hans-Urs von Balthasar (Cosmic Liturgy : the Universe according to Maximus the Confessor, transl. B.E.Daley, San Francisco, Ignatius Press, 2003). Cette liste n'est évidemment pas exhaustive.

${ }_{11}$ Qui est à sa façon, à l'instar du Timée, un modèle explicatif de la constitution du monde.

12 Par exemple : Platon, Timée [37d], A.Rivaud ed. ; Plotin, Enneas III.7 [45], Plotini Opera, T.I, P. Henry et H.-R. Schwyzer eds.; PSEudO-DenYs, Noms divins X.3. [216.2ss], Corpus Dionysiacum I : Pseudo-Dionysius Areopagita. De divinis nominibus, B.R. Suchla ed.
} 
Quant à la présence d'Elie le Prophète, elle serait symboliquement le 'type' de la nature des êtres. Là aussi le moine byzantin en donne la raison. Elie, pour avoir gardé inviolé en lui les raisons de la nature, fut aussi celui qui eut pour charge d'éduquer ceux qui usèrent 'contre-nature' de la nature, eux qui du fait de leur mauvaise inclination penchaient vers le non-être. Elie représente donc en type l'être de la nature dans son intégrité. $^{13}$

Il ne présente que peu d'intérêt de discuter ici la validité de la méthode et la pertinence de ce genre d'exégèse ${ }^{14}$ assez fréquent chez Maxime mais les notions mobilisées et mises en parallèle sont révélatrices d'une certaine vision de la démiurgie qu'il nous peut être utile de déchiffrer.

Apparemment, lorsque Maxime allègue : «Tous ceux qui sont après Dieu, qui sont nés de Dieu, c'est-à-dire la nature des êtres et le temps, apparaissent ensemble auprès de Dieu qui se manifeste réellement, autant qu'il est possible, comme cause et créateur», il ne dit que peu de choses du rapport qui pourrait exister entre la nature des êtres et le temps, sinon deux aspects fondamentaux : ils viennent après Dieu [ $\tau \dot{\alpha} \mu \varepsilon \tau \grave{\alpha}$

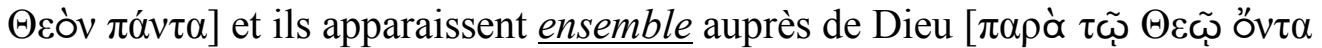

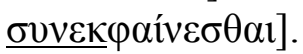

\section{Commençons par trois précisions.}

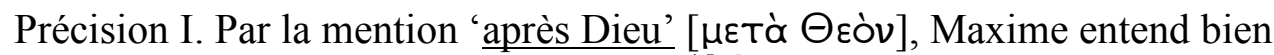
distinguer sans équivoque Créateur et créatures. ${ }^{15}$ À ce titre, il convoque volontiers dans ses écrits de maturité ${ }^{16} 1$ 'opposition 'sans principe' ö $v \propto \rho \chi 0 \varsigma$ ) et de 'pas sans principe' (oưк ớv $\alpha \rho \chi \alpha$ ) pour désigner respectivement le divin seul dont la puissance infinie, cause de tout et circonscrivant tout, étant 'hors catégories', précède métaphysiquement le 'tout' (cf., Ambiguum 10, 1180c) et tout ce qui vient après Dieu.

Précision II. Dans le contexte de cet Ambiguum, 'nature des êtres' embrasse, à la manière du vocable 'Ciel' dans le Timée, ${ }^{17}$ tout ce qui a été, est et sera contenu dans le monde. Il n'est pas invraisemblable que Maxime nous offre par le choix du mot 'nature' une version de platonisme mâtinée d'une forme d'aristotélisme qui lui est propre. Ce choix - pour peu qu'il ait été conscient - lui permettait notamment d'échapper au problème, très sérieux pour la conscience chrétienne, du statut du Ciel. Celui-ci, pour les Néoplatoniciens, ne pouvait avoir commencé et ne pouvait être soumis aux conditions de génération et de corruption du monde sublunaire. ${ }^{18}$

\footnotetext{
${ }^{13}$ Cf., supra toute la partie $\Theta$ ' de la section intitulée 'Théoria sur Moïse et Elie', in : Ambiguum 10, 1164a-1164d, PG 91.

${ }^{14}$ L'un des principaux promoteurs en fut Origène dont les nombreux commentaires bibliques le montre rompu à l'exégèse allégorique.

${ }^{15}$ Il l'avait fait dès les Centuries sur la Charité qui sont, d'après les spécialistes, la première œuvre du moine byzantin. Voir par exemple : MAXIME LE CONFESSEUR, Centuries sur la Charité [III.28 ; IV.6], A.Ceresa-Gastaldo ed.

${ }_{17}$ Parmi lesquels nous comptons les Ambigua ad Iohannem d'où est tiré le texte qui nous intéresse ici.

${ }^{17}$ Cf., Timée [28b, 30d, 31b], A.Rivaud ed., qui connaîtra sur ce point une large postérité. Voir : ARISTOTE, Physique IV [212b17ss], H.Carteron ed. ; JEAN PHILOPON, De opificio mundi [12.8ss], W.Reichardt ed. ; РнотіUs, Bibliothèque, t.V ([cod.240 [323a20ss]), R.Henry ed.

18 Pour comprendre les clés du problème, il faut renvoyer au très bel article de Philippe Hoffmann qui rassemble les principales pièces de ce dossier brûlant que Maxime le Confesseur ne peut avoir ignoré. Voir donc : Ph.HofFMANN, «Sur quelques aspects de la polémique de Simplicius contre Jean Philopon: De l'invective à la réaffirmation de la transcendance du ciel », in: Simplicius, sa vie, son æuvre, sa survie. Actes du colloque international de Paris (28 sept. $-1^{\text {er }}$ oct.1985), I. Hadot ed., Berlin-New York,
} 
Précision III. Rien n'est dit du statut exact du 'temps'. On relève toutefois sa proximité de la nature des êtres avec laquelle il partage le privilège de représenter tous ceux qui venant après Dieu sont nés de Dieu (cf., l'usage par Maxime d'un $\tau$ ov $\tau \varepsilon ́ \sigma \tau \imath$ explicatif).

Dans ce paragraphe, Maxime ne mentionne donc 'nature' et 'temps' qu'en

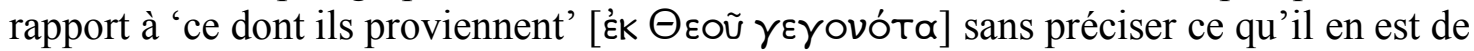
leur rapport mutuel et particulièrement de leur 'statut' à proximité du Démiurge. Toutefois, l'ensemble de l'Ambiguum 10, dans lequel s'inscrit pour Maxime la thèse de la concomitance du temps et de la nature, comporte d'autres éléments en mesure de satisfaire les besoins de notre propos.

\section{Les présupposés de base du système du monde de Maxime le Confesseur}

Dans l'Ambiguum 10, le savant byzantin dévoile un postulat fondamental, passé souvent inaperçu, ${ }^{19}$ de son système :

«Tous les êtres ont de toute évidence pris - à proportion de ce qu'ils sont euxmêmes - l'être 'quand' et l'être 'où'. Sans ceux-ci absolument rien ne pourra être, ni essence, ni quantité, ni qualité, ni relation, ni action, ni passion, ni motion, ni disposition, ni aucune autre parmi les catégories dans lesquelles les

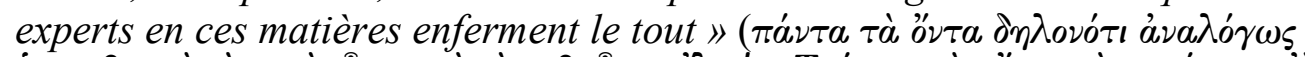

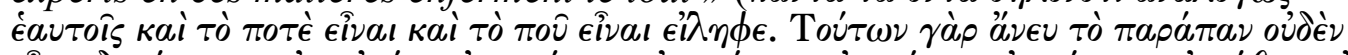

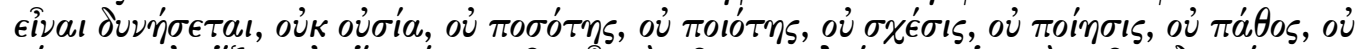

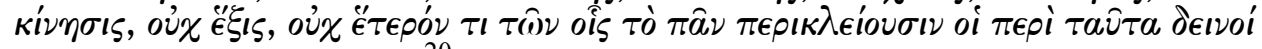
$\left[\right.$ Ambiguum 10, 1181b]). ${ }^{20}$

Par ailleurs, toujours selon le moine byzantin, 'tous les êtres, quels qu'ils soient, ${ }^{21}$ se

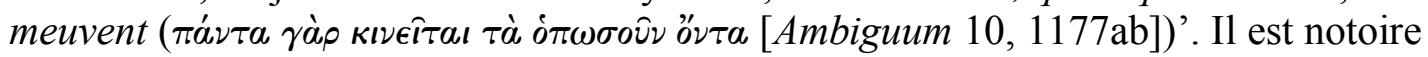
que pour Maxime le Confesseur, à l'instar du Stagirite sur ce point, le mouvement est universel et intrinsèque à la nature de tout ce qui, d'une manière ou d'une autre, vient après le moteur immobile. ${ }^{22}$ S'il est certainement un point qui, dans le soubassement philosophique de la cosmologie maximienne, unit nature et temps, c'est leur rapport

Walter De Gruyter, 1987, p.183-221.

19 C'est d'ailleurs assez curieusement le cas de l'article de Paul Plass, excellent par ailleurs, qui semble méconnaître ce point de doctrine pourtant incontournable de la pensée de Maxime : P.PLASS, « Transcendant Time in Maximus the Confessor », The Thomist 44 (1980) 259-277.

${ }^{20}$ Pour un examen approfondi de cette importante thèse recueillie et reformulée par Maxime, voir les résultats de nos propres travaux rapportés dans notre récente étude : P.MUELLER-JOURDAN, Typologie spatio-temporelle de l'ecclesia byzantine. La Mystagogie de Maxime le Confesseur dans la culture philosophique de l'Antiquité tardive, Leiden, Brill, 2005, pp.42-71.

${ }^{21}$ Même idée mais plus développée dans l'Ambiguum 7 [1072ab], PG 91 : «Les esprits se meuvent de manière spirituelle, les choses sensibles de façon sensible, ou bien en ligne droite, ou en cercle, ou en spirale». Sur les trois 'espèces' du mouvement : Aristote, Physique IV [261b.28], H.Carteron ed. ; Simplicius, In Aristotelis physicorum libros octo commentaria [602.22-23 \& 603.7-8], H.Diels ed. ; Simplicius, In Aristotelis de Caelo commentaria [132. 24-26], J.L.Heiberg ed.

${ }^{22}$ Voir sur ce point le texte explicite du même Ambiguum : «Aucun mouvement n'est sans

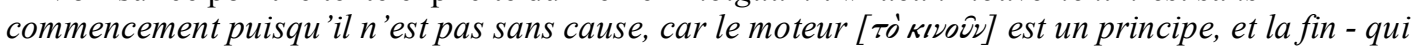
appelle et attire - vers laquelle tout se meut est une cause. Si le moteur est principe de tout mouvement de tout mû, et fin, la cause vers laquelle est porté le mû (rien ne se meut sans cause), aucun des êtres n'est immobile si ce n'est le premier moteur (le premier moteur en effet est immobile [ákíuntov] car aussi sans commencement) ; aucun des êtres donc n'est sans commencement car aucun n'est immobile», in : Ambiguum 10 [1176d-1177a], PG 91. Maxime fait converger dans le même Ambiguum, peut-être sans véritable intention, le Démiurge-artisan du Timée et le Moteur immobile de la Métaphysique. 
respectif au mouvement. La nature est non seulement de l'être en mouvement mais principe de mouvement et de repos ${ }^{23}$ et il est largement admis que le temps en est la mesure. On peut par ailleurs rappeler que le mouvement est la raison invoquée par Timée pour expliquer la naissance du temps (cf., Timée [37cd]) sans qu'il faille dans l'absolu introduire un rapport chronologique autre que celui qu'impose la nature du discours entre l'apparition du mouvement et celui du temps.

La question de savoir si le temps est extrinsèque ou intrinsèque à la nature est dans le cas de Maxime assez délicate à trancher.

Pour Maxime, l'être de la nature, soit l'être en mouvement, n'a de réalité que

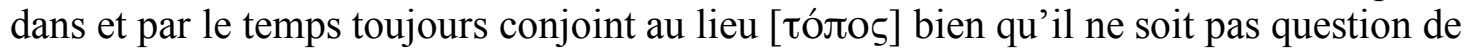
ce dernier dans le contexte de l'apparition de Moïse et d'Elie autour du Verbe Démiurge. On aura noté que sur la liste des catégories adoptées ici par Maxime figure le mouvement, lequel est par conséquent dépendant lui aussi des conditions espacetemps nécessaires à l'existence de l'être des étants.

Il est enfin clair qu'on ne peut introduire du 'chronologique' dans le rapport de succession logique qui découle de ce que l'un, le temps, conjoint au lieu, est dans le cas qui nous intéresse ici, la condition de l'autre. ${ }^{24}$ Comme si le premier avait dû en quelque manière précédé temporellement le second. La priorité du temps, conjoint au lieu, est métaphysique mais, intimement lié à l'être dont il est, avec le lieu, la condition d'existence, il lui est concomittant. Il y a donc simultanéité dans la 'naissance' de la nature comme 'être en mouvement' et du temps.

En bref, pour Maxime, il y a, nés de Dieu, après Dieu et apparaissant ensemble auprès de Dieu, de la nature, du mouvement et une mesure, le temps qui est associé à un nombre qui lui correspond. ${ }^{25}$

\section{Coïncidence du ciel/monde et du temps dans le commentaire de Proclus sur le Timée}

En fonction de la trilogie de notre texte initial, démiurge - nature - temps, il est manifeste que nature et temps apparaissent et, comme nous le verrons ci-après, susbsistent ensemble en conséquence du même et unique acte créateur.

Nous retiendrons ici quelques brèves sections où dans son commentaire sur le Timée Proclus s'attarde à apporter quelques explications au rapport de simultanéité existant entre le temps et le Ciel (ou monde). Nous pensons que nous sommes bien en présence d'un topique similaire à celui que propose Maxime bien que dans le présent cas ce dernier substitue au vocable ciel et/ou monde, le vocable 'nature' à consonnance plus aristotélicienne.

\section{TXT 1}

\footnotetext{
${ }^{23}$ Cf., Aristote, Métaphysique $\Delta, 4$ [1014b16-20], W.D.Ross ed. ; ArISTOTE, Physique I [192b13-16, 20-23], H.Carteron ed. ; ARISTOTE , Physique III [200b12-13], H.Carteron ed. ; voir également chez Maxime : MAXIME LE CONFESSEUR, Opuscules théologiques et polémiques 26, 276a, PG 91.

${ }^{24}$ Il est vrai que sur ce point, nous sommes piégés par les faits de langue et la logique qui découle des mots car il faut que les conditions soient d'abord (!) réunies pour qu'un fait se produise ensuite.

${ }^{25}$ Cf., Ambiguum 10, 1164b, PG 91. Dans le cas qui nous intéresse, il pourrait s'agir simplement du 'décompte' des jours de la création puisque c'est à partir de ce premier comptage du temps que Moïse est dit, selon l'exégèse qu'en fait Maxime, 'type du temps'. Ce décompte revêt dans tous les cas une forme paradigmatique
} 
Livre III. 49.29-50.6, E.Diehl ed. (trad. A.J.Festugière)

[explication de Timée 38b] Les mots «pour que, étant nés ensemble, ils soient ensemble aussi dissous, si jamais ils doivent se dissoudre» montrent clairement que le Ciel est inengendré et impérissable. En effet, s'il est né, c'est dans le Temps qu'il est né. Mais s'il est né «avec le Temps», il n'est pas né dans le Temps : car le Temps non plus n'est pas né dans le Temps, pour qu'il n'y ait pas du Temps avant le Temps. Si donc le Ciel est «né avec le Temps», il n'a pas eu de commencement : car tout ce qui naît doit être postérieur au Temps. Or le Ciel n'est d'aucune façon postérieur au Temps.

\section{TXT 2}

Livre III. 52.9-16, E.Diehl ed. (trad. A.J.Festugière)

[Temps et Ciel se soutiennent mutuellement] Chacun des deux n'est donc pas né pour soi seul ni non plus pour l'autre seul, mais en vue de l'œuvre démiurgique toute entière, pour qu'elle soit rendue le plus possible parfaite et semblable aux modèles - ou plutôt en vue de la bonté du Père de toutes choses, bonté par laquelle la Création aussi atteint son point suprême -. Et une fois devenu ce qu'il est pour l'autre, chacun des deux aide grandement

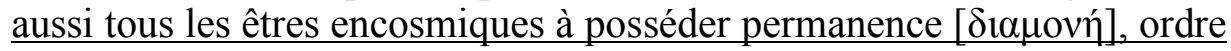
$\left[\tau \alpha^{\prime} \xi_{1 \zeta}\right]$, bon état.

\section{TXT 3}

Livre III. 52.16-33, E.Diehl ed. (trad. A.J.Festugière)

[Temps unique et total] Ici s'achève tout l'exposé philosophique de Platon sur le Temps unique, totale, capable de mesurer toutes choses, qui a été mis en branle et qui fait procession à partir du seul Démiurge et de la monade qui lui est propre. Pour le reste, dans la suite, Platon traitera du Temps tel qu'il se manifeste dans le Ciel et qu'il est comme pluralisé et comme fragmenté en fonction des divers circuits des astres [...] Et quand on voit que les planètes, parmi lesquelles ont été comptés et le soleil et la lune, sont dites avoir été créées pour que ce Temps secondaire se présente visiblement sur la scène, devienne aisément reconnaissable à tous au moyen des astres qui en font voir clairement les mesures partielles et se montre ainsi plus divisé, combien grande ne doit-on pas penser qu'est la dignité qui a été attribuée au Temps tout premier et unique par le Philosophe, ou plutôt par le Démiurge universel lui-même.

\section{TXT 4}

Livre II. 100.1-6, E.Diehl ed. (trad. A.J.Festugière)

[co-subsistance en raison d'un même acte créateur] «Qui doit être à un

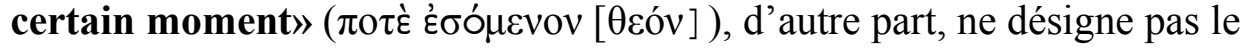


commencement dans le temps, comme le pense Atticus, mais le fait que le Monde a son être coexistant au temps. En effet le temps est né avec le Ciel, et tout à la fois le Monde est dans le temps et le temps est dans le Monde : car ils ont été créés en connexion l'un avec l'autre et ils subsistent ensemble

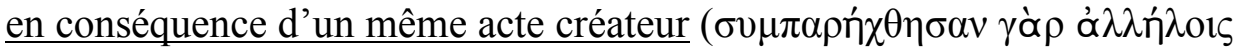

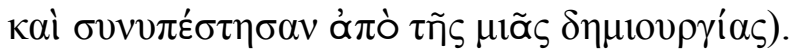

Ce bref sondage dans le Commentaire sur le Timée de Proclus décrit assez clairement, nous semble-t-il, les présupposés en jeu dans l'axiome maximien discuté ici et contribue notamment à élucider le cadre philosophique dans lequel doit être replacé la configuration 'Démiurge - nature - temps'.

\section{Deux sens principaux du vocable 'temps' chez Maxime}

A l'instar des textes de Proclus retenus supra (TXT4), le schéma 'Démiurge nature - temps' est supratemporel et permanent dans le système du moine byzantin et exprime la continuité de l'action providentielle du divin dans l'univers assurant à tous les êtres une permanence, soit la raison de nature qui leur a été assignée, ainsi qu'un ordre ou rang et une position. ${ }^{26}$ Le temps dont il est dès lors question et qui assure dans l'Univers l'ordre ou rang revêt un statut métaphysique affiché et positif qui tranche sur l'expérience intramondaine selon laquelle tout se dissout sous l'effet du temps. ${ }^{27}$ Dans les Centuries sur la théologie et l'économie de l'Incarnation du Fils de Dieu, Maxime,

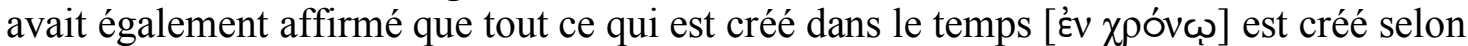

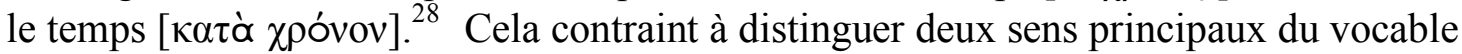
'temps' chez le moine byzantin.

(1) On relève tout d'abord tout comme chez Proclus une sorte de temps premier qui possède un caractère générique. Il est produit comme condition d'existence de l'être

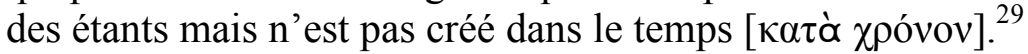

Affirmer le contraire n'aurait pas mis une telle thèse à l'abri de la critique solidement argumentée que Proclus adressait à la doctrine d'Atticus (supra, TXT1, TXT4). Ce paralogisme conduisait à l'impossible création du temps dans le temps ou création temporelle du temps. Doctrine erronée si l'on s'en tient à l'apparition

\footnotetext{
${ }^{26}$ Décrivant les effets de l'agir providentiel divin dans l'Univers, Maxime soutient : «Les saints 'conçurent' d'une part, le bon ordre de la création, la proportion et le commerce que chacun y entretient

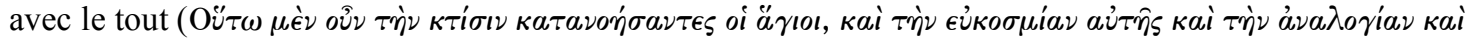

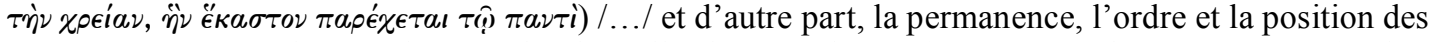
êtres qui y sont nés, leur évolution selon laquelle chacun selon sa forme particulière reste inconfondu et

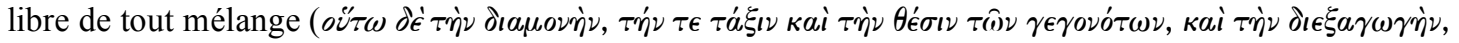

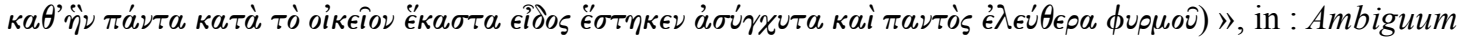
$10,1176 \mathrm{bc}$. Rappelons que ce texte introduit la description maximienne des mouvements intramondains réguliers comme le parcours des astres, le cycle de l'année, l'alternance des jours et des nuits. On se

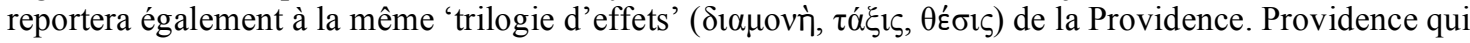
se trouve, affirme Maxime, sur toutes choses. Voir : Ambiguum 10, 1188d, PG 91.

27 Cf., ARIstote, Physique IV [221a30ss], H. Carteron ed.

28 Cf., Centuries sur la théologie et l'économie de l'Incarnation du Fils de Dieu [I.35], 1096c, PG 90.

29 Son rapport à l'activité démiurgique ne peut-être temporelle puisque avant le temps il n'y a pas de temps. Il est par conséquent exclusivement causal.
} 
simultanée du temps et de la nature qui subsistent ensemble en raison d'un même et unique acte créateur. ${ }^{30}$

La thèse d'un temps premier et générique découle donc assez naturellement de l'apparition simultanée de la nature et du temps 'auprès de Dieu' manifesté autant qu'il est possible, avait affirmé Maxime, comme cause et créateur. En bref, on peut admettre dans ce système l'existence d'un temps transcendant, condition sine qua non de l'être et de toutes ses déterminations listées -comme citées précédemment- en 'catégories'. Première et totale expression des logoi du temps qui demeurent en Dieu (la formule est de Maxime, cf., Ambiguum 10, 1164b).

(2) On relève ensuite un temps relatif. Il est, simultanément et grâce au mouvement en train de se faire, objet de perception sensible. Il est relatif aux entités individuées et à leur inévitable succession marquées qu'elles sont par les lois de la génération, de la croissance et de la corruption. Ces dernières sont obligatoirement soumises à une certaine durée et donc produites dans le temps et selon le temps, soit : produites en un certain temps pour disparaître en un certain autre. ${ }^{31}$ Par comparaison au temps premier, ce temps duratif en exprime les principales caractéristiques au niveau des entités individuées à savoir, l'ordre selon l'antérieur et le postérieur et la mesure de leurs mouvements naturels qui peuvent connaître plusieurs variantes ${ }^{32}$ mais qui n'ont qu'un seul ordre, le commencement, le milieu et la fin ${ }^{33}$ soit, plus philosophiquement, de l'antérieur et du postérieur distingués par un certain 'maintenant'.

Comme on l'aura relevé, nous esquivons en partie le problème du statut d'un temps 'autre' qui, si Maxime l'avait traité de façon directe, se classerait comme une variante du temps relatif. Nous voulons en effet faire mention du temps relatif au mouvement du Ciel. Maxime l'avait certes observé ${ }^{34}$ mais ne semble pas avoir tiré de sa régularité la raison de sa perpétuité et ceci parce que, pour le moine byzantin, 'tout

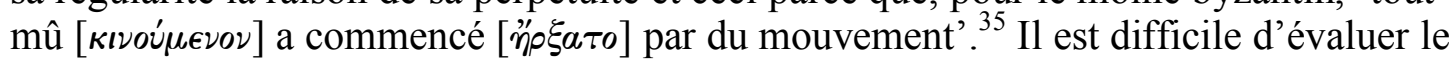
degré de maîtrise que Maxime put avoir de ce délicat dossier mais sa position se rapproche sur ce point de celle que tint Jean Philopon. ${ }^{36}$

\footnotetext{
${ }^{30}$ Cf., Proclus, In Platonis Timaeum Commentaria [II.100.1ss], E.Diehl ed.

31 On perçoit nettement le problème que pourrait poser le statut du monde angélique qui ne tombe évidemment pas sous le coup des lois énoncées ici. Les anges ne sont pas 'sans principe' [oứ ớv $\alpha \rho \chi \alpha$ ].

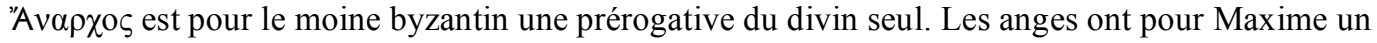
commencement mais ne connaîtront pas de fin. Ils participent toutefois, en raison de leur statut d'êtres créés qui ne fait aucun doute chez notre auteur, au temps intégral qui finalement, toujours chez le savant byzantin, est étroitement associé à l'aiôn (Cf., Centuries sur la théologie et l'économie de l'Incarnation

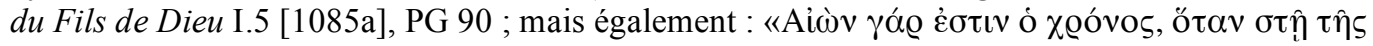

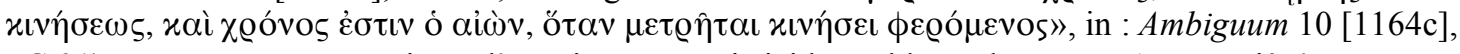
PG 91). Nous sommes conscients d'esquiver en partie ici le problème du rapport 'temps-aiôn'. Nous nous permettons de renvoyer aux observations que nous avons consignées dans l'une de nos précédentes études : P.MuElleR-JOURDAN, Typologie spatio-temporelle de l'ecclesia byzantine. La Mystagogie de Maxime le Confesseur dans la culture philosophique de l'Antiquité tardive, Leiden, Brill, 2005, pp.171172 ; 176-178. On consultera également avec profit : P.PLASS, « Transcendant Time in Maximus the Confessor », The Thomist 44 (1980) 259-277.

32 Cf., Aristote, Physique III [201a.6ss], H.Carteron ed.

33 Cf., MAXIME LE CONFESSEUR, Centuries sur la théologie et l'économie de l'Incarnation du Fils de Dieu I.5 [1085a], PG 90.

34 Sur le mouvement des astres qui toujours se produit de la même manière sans jamais dévier en rien, sur l'égale durée de l'année, sur l'alternance des nuits et des jours, voir : Maxime le Confesseur, Ambiguum 10, $1176 \mathrm{c}$, PG 91.

35 Cf., Ambiguum 10, 1176d-1177a, PG 91.

36 Cf., Jean Philopon, De Aeternitate Mundi contra Proclum, H. Rabe ed., Leipzig, Teubner, 1899 ;
} 
Remarques conclusives:

Le temps et la nature, premiers et dotés de caractères génériques, qui sont le propre du monde conçu dans sa totalité originelle ne peuvent avoir dans le système maximien qu'un statut universel englobant d'innombrables réalisations particulières à savoir l'interpénétration de la nature et du temps dans la réalisation de cet être particulier, de cet autre et de cet autre encore etc. ${ }^{37}$

Il est dès lors vraisemblable que le temps dont Moïse est le 'type', c'est-à-dire l'expression visible et autorisée, est en priorité le temps 'transcendant'. Mais il peut également être, de par la nature même du système maximien, type d'un 'temps' relatif objet de perception sensible- en tant que Moïse reproduit dans son histoire individuelle (naissance - croissance - mort) l'ordre chronologique de toute entité individuée et finalement de ce monde-ci qui se trouve dès lors conçu comme un vivant sensible.

Quant à la nature dont Elie est le type, elle ne peut-être que la nature telle qu'elle fut originellement voulue par le Démiurge soit : dotée d'un certain nombre de déterminations internes qui en constituent la loi. Déterminations qui requièrent, comme nous l'avons signalé dans le postulat fondamental du système maximien, des conditions spatio-temporelles d'existence. Comme expression de l'agir providentiel du Démiurge, la nature est non seulement principe de mouvement et de repos mais surtout le garant d'une conformité et d'une permanence du rapport de tout être passé, présent et futur envers sa raison essentielle. Telle est sans conteste la doctrine de Maxime le Confesseur. $^{38}$

Le temps dont les logoi, soutient Maxime, demeurent en Dieu est non seulement l'expression d'une intention et d'un agir démiurgiques à l'égard de l'être de la nature mais le garant d'un ordre qui s'impose à l'ensemble des êtres qui viennent après Dieu. La notion d'un temps, non seulement ordre ordonné mais ordre ordonnant, expression de la providence - soit de l'action continue du divin dans l'Univers - devait naturellement en découler. Sur ce point, Maxime pouvait s'autoriser d'une déjà longue tradition philosophique qu'on peut faire remonter au Commentaire sur le Timée de Jamblique, commentaire aujourd'hui perdu mais dont Simplicius a consigné plusieurs extraits dans le Corollarium de tempore. ${ }^{39}$

La notion d'un temps, cause de perfection et d'ordre, pouvait être tirée de l'intention démiurgique du Timée [37cd]. Cette compréhension de Platon qu'autorisait, ou même que nécessitait, l'univers fortement hiérarchisé du néoplatonisme en raison de l'extension de l'être 'en mouvement' ${ }^{40}$ devait trancher assez fortement sur l'opinion

voir également : ZACHARIE DE MitYLEne, Ammonius De opificio mundi, PG 85, 1011-1144; la seule édition critique est en langue italienne : Zacaria Scolastico, Ammonio, M. Minniti Colonna, Napoli, 1973.

${ }^{37}$ Un bon exemple en est fourni dans les étapes de développement de l'embryon observées par

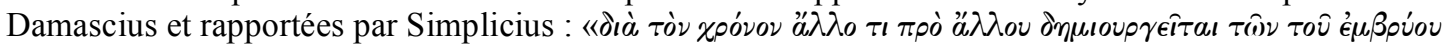

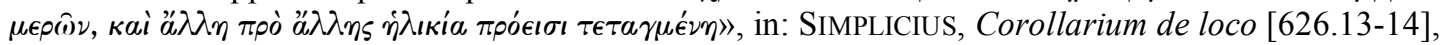
In Aristotelis physicorum libros octo commentaria, H. Diels ed.

38 Cf., Ambiguum 10, 1176bc, PG 91.

39 Voir en particulier : SimPLICIUS, Corollarium de tempore [792.20-795.3], In Aristotelis physicorum libros octo commentaria, H. Diels ed.

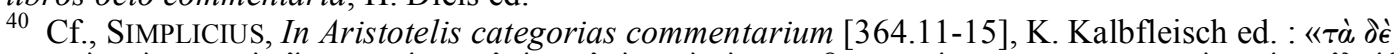

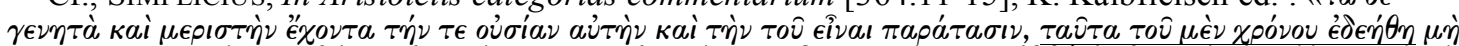

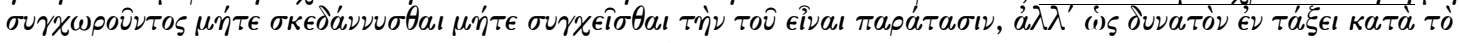

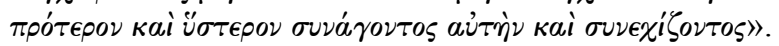


largement admise dans un cadre strictement aristotélicien pour lequel le temps était certes 'mesure du mouvement' mais aussi cause de destruction et d'oubli. Maxime ne nous semble avoir pu ignorer les éléments de ce dossier.

Dans le cas de la manifestation concomittante du temps à celle de la nature des êtres (passé, présent et futur), il en est non seulement la condition sine qua non d'existence mais aussi la limite salutaire, la mesure, présidant 'immobilement' au mouvement de tout ce qui se meut vers la Transfiguration, à terme vers la déification autant qu'il est possible à une nature créée. ${ }^{41}$

\author{
Pascal MUELLER-JOURDAN \\ Université de Fribourg
}

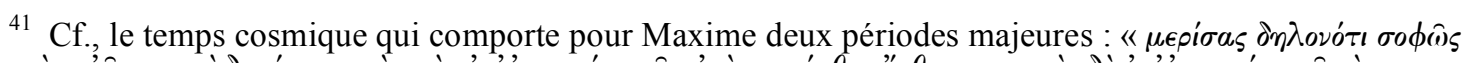

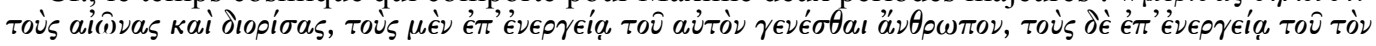

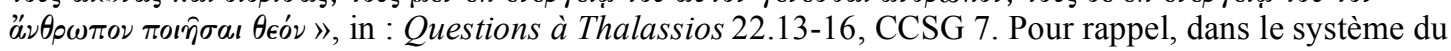
moine byzantin, Dieu a divisé les siècles en deux grandes périodes. Une première partie des siècles appartient au temps de la descente de Dieu vers l'homme, l'autre au temps de l'élévation de l'homme vers Dieu. La première période est vouée à réaliser l'incorporation de Dieu dans l'humanité, la seconde vise à produire la déification de l'homme. 